\title{
Cisplatin/Vinblastine/Cell Penetration Enhancer Formulation INT230-6
}

National Cancer Institute

\section{Source}

National Cancer Institute. Cisplatin/Vinblastine/Cell Penetration Enhancer Formulation

INT230-6. NCI Thesaurus. Code C136428.

A formulation composed of three agents in a fixed ratio: two chemotherapeutic agents, the platinum compound cisplatin and the vinca alkaloid vinblastine, and a proprietary amphiphilic excipient that acts as a penetration enhancer, with potential antineoplastic activity. Upon intra-tumoral (IT) injection of INT230-6, the dispersion/cell penetration enhancer excipient of INT 230-6 facilitates dispersion of the two drugs throughout the tumor tissue and enables increased cellular uptake of these agents into tumor cells. Once inside the cell, cisplatin forms highly reactive, charged, platinum complexes which bind to nucleophilic groups such as GC-rich sites in DNA, which results in apoptosis and cell growth inhibition. Vinblastine kills the tumor cells through binding to tubulin and thereby inhibits microtubule formation, resulting in disruption of the mitotic spindle assembly and cell cycle arrest of tumor. In addition, the tumor cell killing leads to recruitment of dendritic cells (DCs) and induces a tumor-specific T-cell-mediated immune response that attacks both the injected tumor and distant tumor lesions. Local administration of both cisplatin and vinblastine, without the diffusion/penetration enhancer, results in to poor diffusion and a lack of cellular uptake of the agents; INT230-6 increases the intracellular concentration of cisplatin and vinblastine, thereby improving efficacy. 